\title{
Formation of fivefold deformation twins in nanocrystalline face-centered-cubic copper based on molecular dynamics simulations
}

\author{
A. J. $\mathrm{CaO}^{\mathrm{a})}$ and Y. G. Wei ${ }^{\mathrm{b})}$ \\ State Key Laboratory of Nonlinear Mechanics, Institute of Mechanics, Chinese Academy of Sciences, \\ Beijing 100080, People's Republic of China
}

(Received 1 May 2006; accepted 11 June 2006; published online 28 July 2006)

\begin{abstract}
Fivefold deformation twins were reported recently to be observed in the experiment of the nanocrystalline face-centered-cubic metals and alloys. However, they were not predicted previously based on the molecular dynamics (MD) simulations and the reason was thought to be a uniaxial tension considered in the simulations. In the present investigation, through introducing pretwins in grain regions, using the MD simulations, the authors predict out the fivefold deformation twins in the grain regions of the nanocrystal grain cell, which undergoes a uniaxial tension. It is shown in their simulation results that series of Shockley partial dislocations emitted from grain boundaries provide sequential twining mechanism, which results in fivefold deformation twins. (C) 2006 American Institute of Physics. [DOI: 10.1063/1.2243958]
\end{abstract}

Recent studies have shown that the nanocrystalline (nc) materials with the nanosized twin structures have both the high strength and high ductility. In order to explore the advantage mechanical behaviors, the researches on the twiningreinforced mechanism have received the considerable attentions recently. As one kind of the important twiningreinforced mechanisms, the mechanism of the fivefold deformation twins (DTs) has been focused on recently, which has been observed in the experiments of the nanocrystalline face-centered-cubic (fcc) metals and alloys. ${ }^{1,2}$ Previously, no report was given on finding the fivefold deformation twins by using the molecular dynamics (MD) simulations, ${ }^{3-10}$ and it was thought that a uniaxial stress condition used in the MD simulations led to no finding of the fivefold deformation twins. ${ }^{11}$ In the present study, we think that the uniaxial stress condition adopted in the MD simulations is not the main reason because the stress state in the crystal grain cell is always a complex stress state even though a unidirection stress condition is exerted on the boundaries. The object of this letter is to reveal a formation mechanism of fivefold DTs at uniaxial tensile loading state by using the MD simulation, considering the growth pretwins existed in grain regions before the uniaxial loading is exerted.

A textured $\langle 110\rangle$ columnar microstructure is considered here. A representative cell is consisted of four columnar, uniform hexagonal fcc grains that are rotated with respect to each other about the $[1 \overline{1} 0]$ texture (or tilt) axis which defines the $Z$ direction. The nc copper samples are described as that within the grains, some growth twins are introduced, as shown in Fig. 1(a). Specifically, we consider the case that grain size is equal to $11.5 \mathrm{~nm}$, which is comparable to the existed experiment with an average grain size of 10-20 nm. In the present simulation, about 57000 atoms are contained. Figure 1(b) shows the equilibrated configuration at temperature of $300 \mathrm{~K}$ and 0 bar pressure. For comparison, a simulation for no-pretwin sample is also performed.

Similarly to the other research groups, ${ }^{3-10}$ we assigned colors to the atoms according to a local crystallinity classification visualized by common neighbor analysis, ${ }^{12}$ which per-

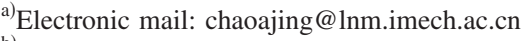

b)Electronic mail: ywei@lnm.imech.ac.cn
}

mit the distinction between atoms in a local hexagonal-closepacked (hcp) environment and those in a fcc environment. Gray stands for fcc atoms, red for hcp atoms, green for other 12-coordinated atoms, and blue for the non-12-coordinated atoms. A single line of hcp atoms represents a twin boundary, two adjacent hcp lines stand for an intrinsic stacking fault, and two hcp lines with a fcc line stand for an extrinsic stacking fault between them.

An empirical Embedded Atom Method (EAM) potential $^{14}$ developed by Daw and Baskes ${ }^{13}$ was used in the present study. The generalized planar fault ${ }^{6}$ energy curves for the stacking and twin fault planar defects are shown in Fig. 2. From Fig. 2, the stacking fault energy $\gamma_{\text {sf }}$ and unstable stacking fault energy $\gamma_{\text {usf }}$ are equal to about 17.2 and $157.8 \mathrm{~mJ} / \mathrm{m}^{2}$, respectively. Unstable twin fault energy $\gamma_{\mathrm{ut}}$ is equal to $166.9 \mathrm{~mJ} / \mathrm{m}^{2}$. These fault energies are of importance in nucleation of dislocations and twins. ${ }^{6}$

The simulated sample is firstly relaxed in the isobaricisothermal ensemble under both the pressure of 0 bar and temperature of $300 \mathrm{~K}$. After equilibration processes, simulation starts under the constant strain rate condition. The equation of motion by Melchionna et al. and Melchionna for the NPT ensemble governs the dynamics of the system. ${ }^{15,16} \mathrm{~A}$ constant strain rate of $1 \times 10^{9} \mathrm{~s}^{-1}$ is adopted in $X$ direction, and both boundary conditions in $Y$ and $Z$ directions are stress-free. Similar method is used in Ref. 10.

Figure 3 shows the typical tensile stress strain curve in strain rate of $1 \times 10^{9} \mathrm{~s}^{-1}$ for both samples with growth
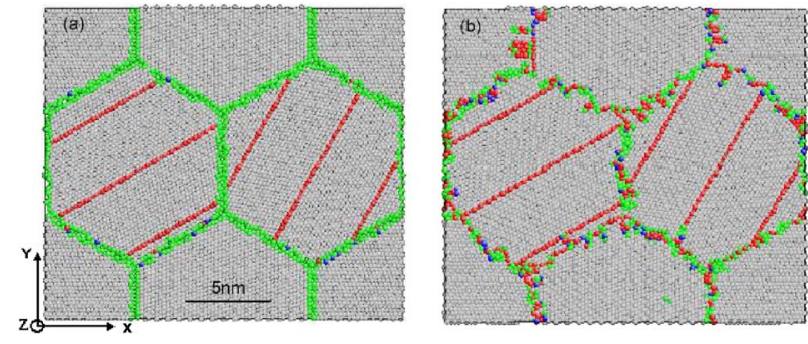

FIG. 1. (Color online) (a) Initial configuration of simulated cell with growth pretwins distributed in some of the grains (b) Relaxed equilibrated configuration at $300 \mathrm{~K}$ and 0 bar pressure. Atoms are colored according to common neighbor analysis. 


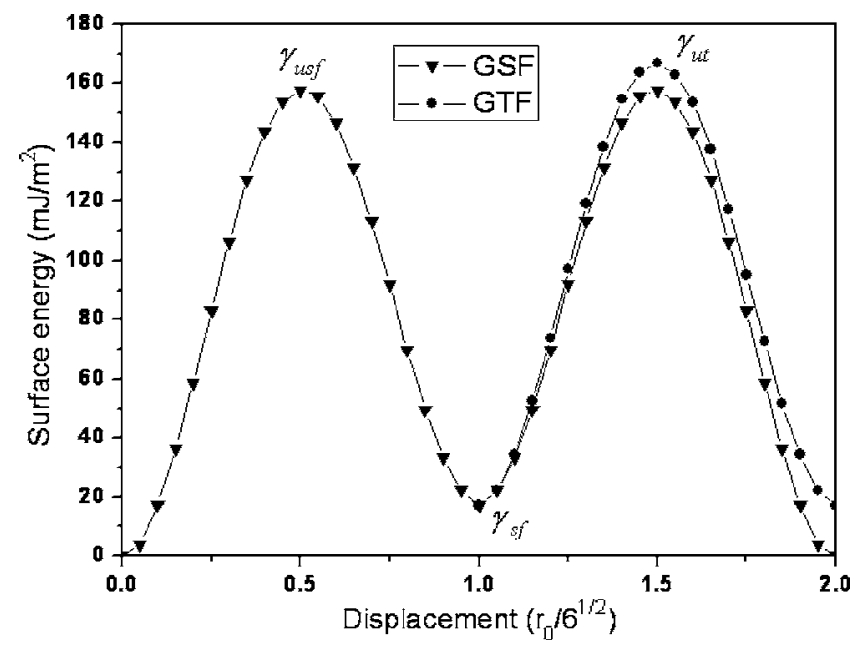

FIG. 2. Curves of the generalized planar fault energy for stacking and twin fault planar defects of copper using the EAM potential of Daw and Baskes (Ref. 13). $\gamma_{\mathrm{sf}}$ is the stable stacking fault energy, $\gamma_{\mathrm{usf}}$ is the unstable stacking fault energy, $\gamma_{\mathrm{ut}}$ is the unstable twin fault energy, and $r_{0}$ is the lattice constant.

pretwins and without pretwins. Elastic Young's moduli are quite the same for two samples, which is about $115 \mathrm{GPa}$. The flow stresses estimated as the average tensile stress after a strain are equal to 3.2 and $2.8 \mathrm{GPa}$ for pretwin and no pretwin samples, respectively. The results imply that the pretwin microstructure causes a higher flow stress.

Figure 4 shows the snapshot of fivefold DT formation process. From Fig. 4(a), the first twin is formed by partial dislocations emission from grain boundaries (GBs) in different grains, and thus the GB1 is transformed into TB1. From Fig. 4(b), a microtwin forms at the GB via emission partial $\mathbf{b}_{1}$, and at the same time a $\mathbf{b}_{2}$ partial glides to the left under the shear stress, which is activated even under a small stress and the twins nucleate via a stress-controlled twin growth mechanism. ${ }^{17,18}$ From Fig. 4(c), threefold twins are formed with another $\mathbf{b}_{3}$ partial emission. In the similar way, a microtwin is formed by series of $\mathbf{b}_{4}$ partials emitted from GBs [see Fig. 4(d)], and then a $\mathbf{b}_{3}$ partial moves away from TB4 glide on the $(11 \overline{1})$ plane and is absorbed at the opposing GBs [see Figs. 4(d) and 4(e)]. Finally, a $\mathbf{b}_{4}$ partial emitted from GBs results in the final fivefold DTs [see Fig. 4(f)]. It is clearly revealed based on the snapshot that the sequential

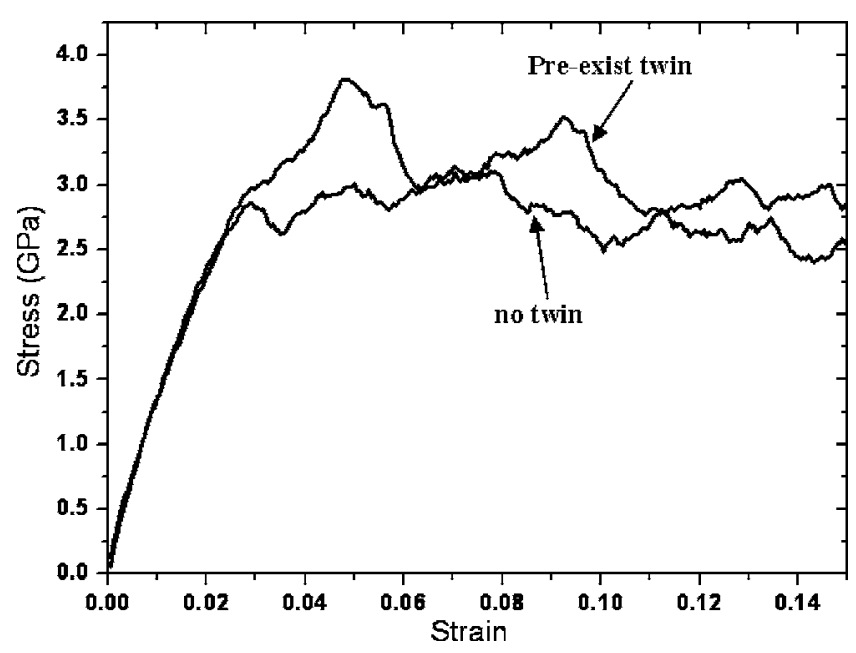

FIG. 3. Stress strain curve for the nc copper samples with and without

growth pretwins under the tensile loading strain rate of $1 \times 10^{9} \mathrm{~s}^{-1}$. uniaxial tensile test with strain rate controlling through intro-
Downloaded 17 Oct 2006 to 159.226 .230 .75 . Redistribution subject to AlP license or copyright, see http://apl.aip.org/apl/copyright.jsp

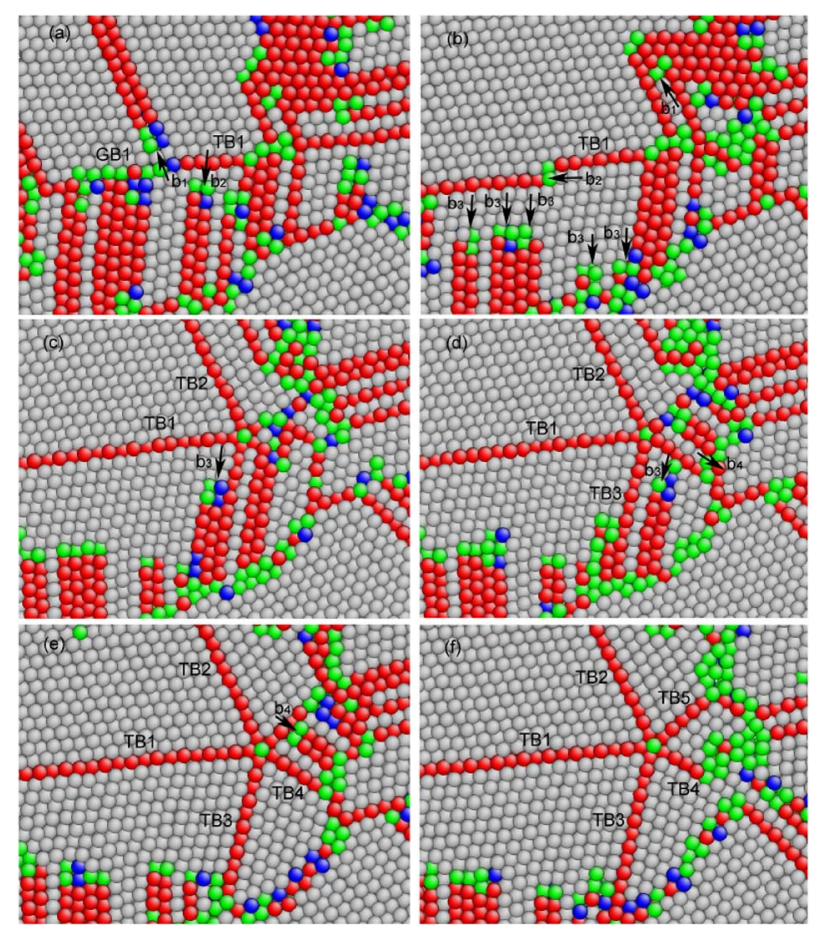

FIG. 4. (Color online) Fivefold DT formation step snapshot. (a) The first

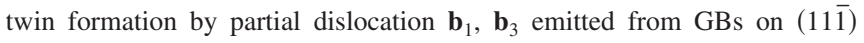
plane in different grains and transform GB1 into TB1; the Burgers of dislocations is $\mathbf{b}_{1}=1 / 6[\overline{1} \overline{1} \overline{2}]$; (b) the second twin is formed by nucleation of a microtwin at the GB, and at the same time a series of partials $\mathbf{b}_{2}, \mathbf{b}_{3}$ emitted from the GBs; (c) threefold twins are formed via emission of partial $\mathbf{b}_{3}$; (d) another microtwin formed by emission partial $\mathbf{b}_{4}$; (e) fivefold twin formed by partial $\mathbf{b}_{4}$ nucleated and propagated toward the GB; (f) final fivefold DT snapshot. (a) $\varepsilon=9.5 \%$, (b) $\varepsilon=10 \%$, (c) $\varepsilon=12.5 \%$, (d) $\varepsilon=13.7 \%$, (e) $\varepsilon$ $=14.3 \%$, and (f) $\varepsilon=15.5 \%$.

twinning mechanism via emission of Shockley partial dislocation from GBs and twin boundaries (TBs) is the fivefold DT formation mechanism.

It is worth noting that in our simulations the dislocation propagation is blocked by the pretwin boundary and the stress in the GBs is built up, which in consequence facilitates twinning. ${ }^{19}$ Consequently the stress in our simulation is much higher than that given in previous work. ${ }^{3-10}$ Fivefold DTs are formed near GBs, which facilitate the nucleation of partial dislocation from the GBs, considering that GBs possess high elastic strain energy and high local stress concentration intensity, which in turn provide additional driving forces for partial dislocation nucleation. In contrast, under the same loading conditions there are not fivefold DTs left in the samples without pretwin.

It was proposed that the critical requirement for forming a fivefold DT was variation in stress orientation. ${ }^{11}$ We think that it is a complicated process to form a fivefold DT because the stress state is always a complex one within a grain region even though a uniaxial stress condition is exerted on the cell boundaries. It is also worth noting that the strain rate sensitivity is another factor needed to be considered in investigating the deformation mechanism of $\mathrm{nc}$ fcc metals, for the higher strain rate results relatively higher flow stress under all other same conditions. ${ }^{10}$

In summary, we have demonstrated that the fivefold DTs can be formed by sequentially emitting partial dislocations from GBs and TBs by using MD simulation. Such a mechanism can be predicted by using the MD simulation for a uniaxial tensile test with strain rate controlling through introto AIP license or copyright, see http://apl.aip.org/apl/copyright.jsp 
ducing some pretwins within the grain regions. It has noted that a uniaxial stress condition can induce a complex stress state in the interior of the nc grains in the cell due to the nonuniformity and different crystallographic orientation in the grains. Therefore, the true critical requirement and conditions for formation of the fivefold DTs still need to be explored. Through the present investigation, the following factors may take effects on the formation of the fivefold DTs: (1) microstructure features, such as the selected columnar GB dislocation structure, growth pretwins, etc., which may play an important role in plastic deformation; (2) high strain rate, as it is well known that twinning is favored with high strain rate and low temperature; (3) large plastic strain; and (4) loading conditions, such as the constant strain rate controlling and the constant stress level controlling in uniaxial tensile test. The former can cause a stress state more deviated from a uniform stress state within the grain region. The necessity and the importance of these factors still need to be investigated further.

This work is supported by the National Science Foundation of China through Grant Nos. 10432050 and 10428207.
${ }^{1}$ X. Z. Liao, Y. H. Zhao, S. G. Srinivasan, Y. T. Zhu, R. Z. Valiev, and D. V. Gunderov, Appl. Phys. Lett. 84, 592 (2004).

${ }^{2}$ J. Y. Huang, Y. K. Wu, and H. Q. Ye, Acta Mater. 44, 1211 (1996).

${ }^{3}$ V. Yamakov, D. Wolf, S. R. Phillpot, A. K. Mukherjee, and H. Gleiter, Nat. Mater. 1, 1 (2002).

${ }^{4}$ V. Yamakov, D. Wolf, S. R. Phillpot, and H. Gleiter, Acta Mater. 50, 5005 (2002).

${ }^{5}$ H. Van Swygenhoven, Science 296, 66 (2002).

${ }^{6}$ H. Van Swygenhoven, P. M. Derlet, and A. G. Frøseth, Nat. Mater. 3, 399 (2004).

${ }^{7}$ A. G. Frøseth, P. M. Derlet, and H. Van Swygenhoven, Appl. Phys. Lett. 85, 5863 (2004).

${ }^{8}$ A. Froseth, H. Van Swygenhoven, and P. M. Derlet, Acta Mater. 52, 2259 (2004).

${ }^{9}$ J. Schiøtz and K. W. Jacobsen, Science 301, 1357 (2003).

${ }^{10}$ J. Schiøtz, Scr. Mater. 51, 837 (2004).

${ }^{11}$ Y. T. Zhu, X. Z. Liao, and R. Z. Valiev, Appl. Phys. Lett. 86, 103112 (2005).

${ }^{12}$ J. D. Honeycutt and H. C. Andersen, J. Phys. Chem. 91, 4950 (1987).

${ }^{13}$ M. S. Daw and M. I. Baskes, Phys. Rev. B 29, 6443 (1984).

${ }^{14}$ S. M. Foiles, M. S. Daw, and M. I. Baskes, Phys. Rev. B 33, 7983 (1986).

${ }^{15}$ S. Melchionna, G. Ciccotti, and B. L. Holian, Mol. Phys. 78, 533 (1993).

${ }^{16}$ S. Melchionna, Phys. Rev. E 61, 6165 (2000).

${ }^{17}$ Y. T. Zhu, X. Z. Liao, Y. H. Zhao, S. G. Srinivasan, F. Zhou, and E. J. Lavernia, Appl. Phys. Lett. 85, 5049 (2004).

${ }^{18}$ Y. T. Zhu, X. Z. Liao, S. G. Srinivasan, E. J. Lavernia, and R. Z. Valiev, J. Appl. Phys. 98, 034319 (2005).

${ }^{19}$ L. Lu, Y. Shen, X. Chen, L. Qian, and K. Lu, Science 304, 422 (2004). 